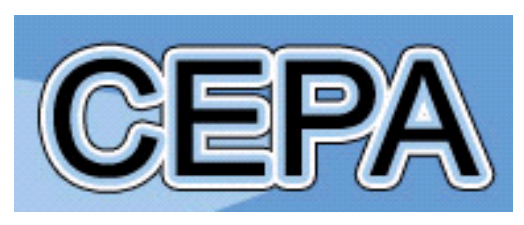

Centre for Efficiency and Productivity Analysis

Working Paper Series

No. WP09/2019

Hicks Neutrality and Homotheticity in Technologies with

Multiple Inputs and Multiple Outputs

Rolf Färe, Hideyuki Mizobuchi, Valentin Zelenyuk

Date: July 2019

\author{
School of Economics \\ University of Queensland \\ St. Lucia, Qld. 4072 \\ Australia
}

ISSN No. 1932 - 4398 


\title{
Hicks Neutrality and Homotheticity in Technologies with Multiple Inputs and Multiple Outputs
}

\author{
Rolf Färe $^{\dagger}$, Hideyuki Mizobuchi ${ }^{\ddagger}$, Valentin Zelenyuk ${ }^{\S}$
}

July 2019

\begin{abstract}
We analyse two popular notions in production theory: the notion of Hicks neutral technical change and the notion of homothetic technology. Both of these notions are characterized by the radial expansions or contractions of the relevant isoquants, yet in different ways. In the case of multiple-input and multiple-output, an isoquant is characterized by either the input or output combinations. Thus, for each of these notions, there are two types of conditions: one is based on the input isoquants and the other is based on the output isoquants. We show that for each notion, these two conditions are equivalent under the $\alpha$-returns to scale technology. From this result, we also derive several implications for the properties of well-known productivity indexes, such as the Malmquist productivity index and the Hicks-Moorsteen productivity index.
\end{abstract}

Keywords: homotheticity, input homotheticity, output homotheticity, inverse homotheticity, $\alpha$-returns to scale technology, Malmquist productivity index, HicksMoorsteen productivity index

JEL classification: C43, D24, L11 O33

\footnotetext{
${ }^{\dagger}$ Department of Economics, Oregon State University and Department of Agricultural and Resource Economics, University of Maryland, USA, rolf.fare@ orst.edu.

${ }^{\ddagger}$ Faculty of Economics, Ryukoku University, 67 Fukakusa Tsukamoto-cho, Fushimi-ku, Kyoto 6128577, Japan, mizobuchi@econ.ryukoku.ac.jp.

${ }^{\S}$ School of Economics and Centre for Efficiency and Productivity Analysis, The University of Queensland, 530, Colin Clark Building (39), St. Lucia, Brisbane, QLD 4072, Australia; v.zelenyuk@uq.edu.au.
} 


\section{Introduction}

Homotheticity and Hicks neutrality are concepts that have been widely used in a variety of fields of economics. Homotheticity is a property of the technology of a firm, which implies a separability between inputs and outputs. On the other hand, Hicks neutrality is a property of the technology change of a firm, which implies a separability of this change from the inputs or outputs or both. While they are different concepts, they share one aspect: both of them are characterized by either the input or output isoquants.

Shephard (1953) introduced the notion of a homothetic production function as the increasing transformation of the homogeneous function of factor inputs in the single output case. The input isoquant radially expands or contracts as output changes under this function. This idea was generalized to the multiple output case by Shephard (1970). ${ }^{1}$ In the multiple output case, there is the output isoquant as well as the input isoquant. Thus, two types of homotheticity are considered. One is input homotheticity, which requires the input isoquant to expand or contract radially, and the other is output homotheticity, which requires the output isoquant to expand or contract radially.

Hicks (1932) introduced the notion of Hicks neutral technical change for the single output case. Under this type of technical change, the input isoquant contracts or expands radially with time and thus, the marginal rate of substitution between any pairs of inputs is independent of time. As Chambers and Färe (1994) show in their formal taxonomy of the various types of Hicks neutrality, there are also two types of Hicks neutral technical change in the multiple output case. One is Hicks input neutral technical change, which induces the input isoquant to contract or expand radially, and the other is Hicks output neutral technical change, which induces the output isoquant to expand or contract radially. ${ }^{2}$

Homotheticity and Hicks neutrality play an important theoretical role especially in index number theory. ${ }^{3}$ These conditions imply the separability of distance, cost or revenue functions in input and output quantities, which make the price and quantity index independent of the reference price and quantity vector. ${ }^{4}$ They also imply the equivalence between two productivity indexes such as Malmquist and Hicks-Moorsteen indexes. $^{5}$

The input-isoquant-based condition such as input homotheticity and Hicks input neutrality is sometimes imposed alone and sometimes imposed along with the outputisoquant-based condition such as output homotheticity and Hicks output neutrality, and some returns to scale conditions. However, it appears that the relationship between the input-isoquant-based condition and the output-isoquant-based condition has not been explored.

\footnotetext{
${ }^{1}$ Shephard (1970) refers to Jacobsen (1970) as the study using the same definition.

${ }^{2}$ Sato and Ramachandran (1998) examine the 'holothetic' technology where the more general type of technical change can be regarded as the mapping of isoquants. Here, we focus on the technology and the technical change which are characterized by the radial expansion of isoquants along with the input and output change as well as time.

${ }^{3}$ For example, see Färe and Primont (1995) and Sickles and Zelenyuk (2018).

${ }^{4}$ See Shephard (1970), Färe and Mitchell (1993), Färe and Primont (1995), and Balk (1998) for the implication of homotheticity. See Chambers and Färe (1994) for the implication of Hicks neutrality.

${ }^{5}$ Färe et al. (1996) and Balk (1998) show that these two indexes are equivalent when we assume homothetic technology. Mizobuchi (2017b) show that these two indexes are equivalent when we assume Hicks neutral technical change.
} 
In this paper, we examine the type of technology under which two types of conditions are equivalent. The $\alpha$-returns to scale technology (which is firstly proposed by Lau (1978) and explored by Färe and Mitchell (1993) and more recently by Boussemart et al. (2009)) is known as the general concept of returns to scale that incorporates different degrees of returns to scale. Here, we show that when the technology satisfies $\alpha$-returns to scale, input and output homothetic technologies are equivalent and Hicks input and output technical changes are also equivalent. These interesting theoretical facts appear to be new to the literature on production theory.

The remainder of this paper is organised as follows. Section 2 explains the model of the firm this paper relies on and introduces the definitions of the theoretical productivity indexes. Section 3 introduces the concepts of homothetic technology and Hicks neutral technical change. Section 4 deals with the $\alpha$-returns to scale technology. Section 5 includes the main results. Finally, Section 6 concludes the paper.

\section{Technology Characterizations and Theoretical Productivity Indexes}

Consider a firm that produces $M$ types of outputs $y=\left(y_{1}, \ldots, y_{M}\right) \in \mathbb{R}_{+}^{M}$ from $N$ types of inputs $x=\left(x_{1}, \ldots, x_{N}\right) \in \mathbb{R}_{+}^{N}$. Let $t \in \tau$ indicate a particular time period in a relevant range of time denoted by $\tau .{ }^{6}$ The firm's technology available at a period $t$ is fully characterized by the technology set $T^{t}$, which is the set of all feasible combinations of inputs and outputs, defined as:

$$
T^{t} \equiv\left\{(x, y) \in \mathbb{R}_{+}^{N+M}: x \text { can produce } y \text { in period } t\right\}
$$

The production technology $T^{t}$ can be equivalently represented via the output set $P^{t}(x)$, defined as

$$
P^{t}(x) \equiv\left\{y \in \mathbb{R}_{+}^{M}:(x, y) \in T^{t}\right\}
$$

and the input set $L^{t}(y)$, defined as

$$
L^{t}(y) \equiv\left\{x \in \mathbb{R}_{+}^{N}:(x, y) \in T^{t}\right\}
$$

We assume that the technology satisfies the following regularity conditions: ${ }^{7}$ (T.1) no free lunch: $y \notin P^{t}\left(0_{N}\right)$ for any $y \in \mathbb{R}_{+}^{M}$; (T.2) zero production is possible with any input: $0_{M} \in P^{t}(x)$ for any $x \in \mathbb{R}_{+}^{N}$; (T.3) strong disposability of outputs: $y \in P^{t}(x)$ and $y^{*} \leq y$ implies $y^{*} \in P^{t}(x)$ for any $x \in \mathbb{R}_{+}^{N}$; (T.4) strong disposability of inputs: $x \in L^{t}(y)$ and $x^{*} \geq x$ implies $x^{*} \in L^{t}(y)$ for any $y \in \mathbb{R}_{+}^{M}$; (T.5) $T^{t}$ is closed and (T.6) $P^{t}(x)$ is bounded.

\footnotetext{
${ }^{6}$ In general, $\tau$ can be $\mathbb{R}_{+}$or a subset of it or a set of discrete values of interest.

${ }^{7}$ Vector notation: $y \geq y^{\prime}$ indicates $y_{m} \geq y_{m}^{\prime}$ for any $m ; y \gg y^{\prime}$ indicates $y_{m}>y_{m}^{\prime}$ for any $m ; y>y^{\prime}$ indicates $y_{m} \geq y_{m}^{\prime}$ for any $m$ and $y \neq y^{\prime}$; and $0_{M}$ and $1_{M}$ denotes $M$ dimensional vector of zeros and ones, respectively.
} 
These conventional axioms on the technology guarantee the existence of well-defined distance functions that completely (i.e., equivalently) characterize the technology defined in (1) or (2) or (3) (Färe and Primont, 1995). In particular, the period $t$ output distance function $D_{o}^{t}: \mathbb{R}_{+}^{N+M} \rightarrow \mathbb{R}_{+} \cup\{+\infty\}$ characterizes the technology of period $t$ and is defined as

$$
D_{o}^{t}(x, y) \equiv \inf \left\{\theta>0:(x, y / \theta) \in T^{t}\right\}
$$

Meanwhile, the period $t$ input distance function $D_{i}^{t}: \mathbb{R}_{+}^{M+N} \rightarrow \mathbb{R}_{+} \cup\{+\infty\}$ also characterizes the technology of period $t$ and is defined as:

$$
D_{i}^{t}(y, x) \equiv \sup \left\{\theta>0:(x / \theta, y) \in T^{t}\right\}
$$

These distance functions are convenient tools for describing the underlying technology. In particular, the fact that a production plan $(x, y)$ is feasible at the period $t$ technology such as $(x, y) \in T^{t}$ is equivalent to saying $0 \leq D_{o}^{t}(x, y) \leq 1$ or $D_{i}^{t}(y, x) \geq 1$, which is known as the complete characterization property of these functions. ${ }^{8}$

Having the characterization property, makes the distance functions useful tools for defining various theoretical indexes, e.g. Malmquist and Hicks-Moorsteen productivity indexes, which appear to be the most popular theoretical productivity indexes. ${ }^{9}$

In particular, recall that the Malmquist productivity index, which was first introduced by Caves et al. (1982), compares the radial distances of input and output vectors, in some periods of interest denoted by 0 and 1 (elements in $\tau$ ), relative to the reference technology. The output-oriented Malmquist productivity index with respect to the period $t$ technology $M P I^{t}$ is defined as follows:

$$
M P I^{t} \equiv \frac{D_{o}^{t}\left(x^{1}, y^{1}\right)}{D_{o}^{t}\left(x^{0}, y^{0}\right)}
$$

We simply call MPI ${ }^{t}$ the period $t$ Malmquist productivity index. The technologies of the periods 0 and 1 can be considered as equally reasonable candidates of the reference technology for measuring productivity growth between periods 0 and 1 , leading to two indexes, $M P I^{0}$ and $M P I^{1}$. In order to reconcile between them, Caves et al. (1982) define the Malmquist productivity index MPI by their geometric mean as follows:

$$
M P I \equiv \sqrt{M P I^{0} \times M P I^{1}}
$$

\footnotetext{
${ }^{8}$ Hereafter we will restrict our focus on $D_{o}^{t}(x, y) \in(0,+\infty)$ or $D_{i}^{t}(y, x) \in(0,+\infty)$ for all $(x, y) \in$ $\mathbb{R}_{+}^{N+M}$, so that they yield finite values when used in the indexes.

${ }^{9}$ Another popular theoretical measure of productivity growth is the Luenberger productivity indicator introduced by Chambers (2002). See Briec and Kerstens (2004) and Balk et al. (2008) for the relationship between the Luenberger productivity indicator and the Malmquist productivity indicator.
} 
The Hicks-Moorsteen productivity index, which is first envisioned by Diewert (1992) and later formulated by Bjurek (1996), compares the growth rate of outputs and that of inputs in periods 0 and 1. Each growth rate is measured by the Malmquist output and input quantity indexes, which compare the radial distances of output vectors and input vectors in periods 0 and 1 relative to the reference technology. The Hicks-Moorsteen productivity index with respect to the period $t$ technology $H M P I^{t}$ is defined as follows:

$$
H M P I^{t} \equiv \frac{D_{o}^{t}\left(x^{t}, y^{1}\right) / D_{o}^{t}\left(x^{t}, y^{0}\right)}{D_{i}^{t}\left(y^{t}, x^{1}\right) / D_{i}^{t}\left(y^{t}, x^{0}\right)}
$$

We simply call $H M P I^{t}$ the period $t$ Hicks-Moorsteen productivity index. Similarly as for the Malmquist productivity index, the technologies of the periods 0 and 1 can be considered as equally reasonable candidates of the reference technology for measuring productivity growth between periods 0 and 1 , leading to two indexes, $H M P I^{0}$ and $H M P I^{1}$. To reconcile between them, Bjurek (1996) defines the Hicks-Moorsteen productivity index HMPI by their geometric mean as follows: ${ }^{10}$

$$
H M P I \equiv \sqrt{H M P I^{0} \times H M P I^{1}}
$$

\section{Homotheticity and Hicks Neutrality}

In the case of multiple inputs and multiple outputs, there exist several types of homotheticity. Here, we will focus on two types: input and output homotheticity. ${ }^{11}$

Definition 1: The technology is input homothetic ${ }^{12}$ if and only if

$$
L^{t}(y)=H^{t}(y) L^{t}\left(1_{M}\right), \quad \forall y \in \mathbb{R}_{+}^{M},
$$

where $H^{t}: \mathbb{R}_{+}^{M} \rightarrow \mathbb{R}_{++}$is a function that has the following properties consistent with the regularity conditions T.1-T. $6^{13}$ and $1_{M}=(1, \ldots, 1)$ is a constant input vector: ${ }^{14}$ (A.1) $H^{t}\left(0_{M}\right)=0$; (A.2) $H^{t}(y)>0$ if $y>0_{M}$; (A.3) $H^{t}\left(y^{\prime}\right) \geq H^{t}(y)$ if $y^{\prime} \geq y$; (A.4) $H^{t}(y) \rightarrow+\infty$ as $\{\|y\|\} \rightarrow+\infty$; and (A.5) $H^{t}(y)$ is lower semi-continuous.

\footnotetext{
${ }^{10}$ Bjurek (1996) uses the term 'Malmquist total factor productivity index' rather than 'Hicks-Moorsteen productivity index'.

${ }^{11}$ Shephard (1970) first provides the formal definitions of the input and output homotheticity in the multiple output case, which Färe and Mitchell (1993) generalize into a more tractable form. In this paper, we adopt the definition by the latter.

${ }^{12}$ The constant output vector $1_{M}=(1, \ldots, 1)$ can be replaced by an arbitrary output vector $\bar{y}$.

${ }^{13}$ Shephard (1970) points out that $H^{t}(y)$ has these properties: Moreover, $H^{t}(y)$ turns out to satisfy linear homogeneity in the case where the technology exhibits constant returns to scale. These properties guarantee that the input distance function $D_{i}^{t}(y, x)$ defined by Eq. (5) satisfies the properties consistent with the regularity conditions T.1-T.6.

${ }^{14}$ Input distance function $D_{i}^{t}(1, x)$ can be $D_{i}^{t}(\bar{y}, x)$ for arbitrary output vector $\bar{y}$.
} 
In terms of the input distance function, one can alternatively define input homotheticity change as follows: ${ }^{15}$

$$
D_{i}^{t}(y, x)=\frac{D_{i}^{t}\left(1_{M}, x\right)}{H^{t}(y)}, \quad \forall(x, y) \in \mathbb{R}_{+}^{N+M}
$$

Thus, under input homotheticity, each input isoquant can be considered as a radial expansion (or contraction) of the reference input isoquant which corresponds to the unit output. It is noteworthy that the radial distance between each input isoquant and the reference input isoquant is constant regardless of outputs.

Definition 2: The technology is output homothetic ${ }^{16}$ if and only if

$$
P^{t}(x)=G^{t}(x) P^{t}\left(1_{N}\right), \quad \forall x \in \mathbb{R}_{+}^{N},
$$

where $G^{t}: \mathbb{R}_{+}^{N} \rightarrow \mathbb{R}_{++}$is a non-decreasing function that has the following properties consistent with the regularity conditions T.1-T. $6^{17}$ and $1_{N}=(1, \ldots, 1)$ is the unit input vector: (B.1) $G^{t}\left(0_{N}\right)=0$; (B.2) $G^{t}(x)$ is finite for $x \in \mathbb{R}_{+}^{N}$; (B.3) $G^{t}\left(x^{\prime}\right) \geq G^{t}(x)$ if $x^{\prime} \geq x$; (B.4) for any $x \geq 0_{N}$ such that $G^{t}(\lambda x)>0$ for some scalar $\lambda, G^{t}(\lambda x) \rightarrow+\infty$ as $\lambda \rightarrow+\infty$; and (B.5) $G^{t}(x)$ is upper semi-continuous.

By using the output distance function, we can alternatively define output homotheticity change as follows: ${ }^{18}$

$$
D_{o}^{t}(x, y)=\frac{D_{o}^{t}\left(1_{N}, y\right)}{G^{t}(x)}, \quad \forall(x, y) \in \mathbb{R}_{+}^{N+M}
$$

Thus, under the output homotheticity, each output isoquant can be considered as a radial expansion (or contraction) of the reference output isoquant which corresponds to the unit input. It is noteworthy that the radial distance between each output isoquant and the reference output isoquant is constant regardless of inputs.

The following more stringent homotheticity condition goes back to Shephard (1970). It is defined by employing the distance functions directly.

Definition 3: The technology is inverse homothetic if and only if

\footnotetext{
${ }^{15}$ Färe and Mitchell (1993) first show that these conditions of Eqs. (10) and (11) are equivalent.

${ }^{16}$ The constant input vector $1_{N}=(1, \ldots, 1)$ can also be replaced by an arbitrary input vector $\bar{x}$.

${ }^{17}$ Shephard (1970) points out that $G^{t}(x)$ has these properties. Moreover, $G^{t}(x)$ turns out to satisfy linear homogeneity in the case where the technology exhibits constant returns to scale. These properties guarantee that the output distance function $D_{o}^{t}(x, y)$ defined by Eq. (4) satisfies the properties consistent with the regularity conditions T.1-T.6.

${ }^{18}$ Färe and Primont (1995) first show that these conditions of Eqs. (12) and (13) are equivalent.
} 


$$
D_{o}^{t}(x, y)=D_{o}^{t}\left(1_{N}, y\right) / J\left(D_{i}^{t}\left(1_{M}, x\right)\right), \quad \forall(x, y) \in \mathbb{R}_{+}^{N+M},
$$

where $J: \mathbb{R}_{+} \rightarrow \mathbb{R}_{++}$is a continuous and strictly increasing function consistent with the regularity conditions T.1-T.6.

While it is not straightforward to derive implications on the underlying technology from the above definition, Färe and Primont (1995) show that it is equivalent to joint input and output homotheticity. Thus, this homotheticity condition imposes that each input and output isoquants are radial expansions of the reference isoquants.

Proposition 1 (Färe and Primont, 1995): The technology is simultaneously input and output homothetic if and only if it is inverse homothetic.

As the following Propositions suggest, it is known that two popular productivity indexes coincide under the homothetic conditions. It is worth noting that the constant returns to scale is also assumed along with this homotheticity condition.

Proposition 2 (Färe, Grosskopf and Roos 1996): The period $t$ Malmquist productivity index $M P I^{t}$ equals the period $t$ Hicks-Moorsteen productivity index $H M P I^{t}$ if and only if the technology is inversely homothetic and exhibits constant returns to scale.

Proposition 3 (Balk 1998): The Malmquist productivity index MPI equals the HicksMoorsteen productivity index HMPI if the technology is output homothetic and exhibits constant returns to scale.

Meanwhile, the notion of Hicks neutral technical change is also characterized by the radial expansion of isoquants over time. In particular, in the case of multiple outputs, there exist two types of Hicks neutrality conditions. Each condition corresponds to either input or output isoquant. First, we introduce the definition of the Hicks input neutral technical change as the radial expansion of the input isoquant.

Definition 4: ${ }^{19}$ Technical change is Hicks input neutral for the time range $\tau$ if and only if

$$
L^{t}(y)=\frac{\bar{L}(y)}{A(t)}, \quad \forall y \in \mathbb{R}_{+}^{M},
$$

where $A: \tau \rightarrow \mathbb{R}_{++}$is a function consistent with the regularity conditions T.1-T.6.

\footnotetext{
${ }^{19}$ More generally, the implicit Hicks input neutral technical change, which is defined by $L^{t}(y)=$ $\bar{L}(y) / A(t, y)$, allows a factor parameter $A$ to vary with $t$ and $y$. See Blackorby et al. (1976) and Chambers and Färe (1994) for different types of Hicks neutrality.
} 
By using the input distance function, we can obtain the implication of the Hicks input neutral technical change neutrality as follows:

$$
D_{i}^{t}(y, x)=A(t) \bar{D}_{i}(y, x), \quad \forall(x, y) \in \mathbb{R}_{+}^{N+M},
$$

where $\bar{D}_{i}(y, x) \equiv \sup \{\theta>0: x / \theta \in \bar{L}(y)\}$.

Similarly, we introduce the definition of the Hicks output neutral technical change as the radial expansion of the output isoquant.

Definition 5: ${ }^{20}$ Technical change is Hicks output neutral for the time range $\tau$ if and only if

$$
P^{t}(x)=B(t) \bar{P}(x), \quad \forall x \in \mathbb{R}_{+}^{N},
$$

where $B: \tau \rightarrow \mathbb{R}_{++}$is a function consistent with the regularity conditions T.1-T.6.

By using the output distance function, we obtain the implication of the Hicks output neutral technical change as follows:

$$
D_{o}^{t}(x, y)=\frac{\bar{D}_{o}(x, y)}{B(t)}, \quad \forall(x, y) \in \mathbb{R}_{+}^{N+M},
$$

where $\bar{D}_{o}(x, y) \equiv \inf \{\theta>0: y / \theta \in \bar{P}(x)\}$.

As the following Proposition suggests, it is known that the two popular productivity indexes also coincide under the Hicks neutral technical change. It is worth noting that the constant returns to scale is often assumed, along with the Hicks neutrality condition, to achieve some theoretical results.

Proposition 4 (Mizobuchi, 2017b): The Malmquist productivity index MPI equals the Hicks-Moorsteen productivity index HMPI if the technology exhibits constant returns to scale and the technical change is Hicks output neutral.

\section{Homogenous Technology}

As we outlined in the previous section, there are two types of conditions for the homothetic technology as well as the Hicks neutral technical change. One is represented by the radial expansion of the input isoquants along with output change or time, and the other is represented by the radial expansion of the output isoquants along with input change or time. In this paper, we explore the relationship between the two types of conditions. In particular, we examine when these two conditions become equivalent.

\footnotetext{
${ }^{20}$ More generally, the implicit Hicks output neutral technical change, which is defined by $P^{t}(x)=$ $B(t, x) \bar{P}(x)$, allows a factor parameter $B$ to vary with $t$ and $x$.
} 
The following $\alpha$-returns to scale technology is a general concept of returns to scale that can deal with different degrees of scale. ${ }^{21}$ While the parameter $\alpha$ indicates the degree of returns to scale, it can be chosen freely.

Definition 6: The technology satisfies $\alpha$-returns to scale if and only if there is a scaler $\alpha \in \mathbb{R}$ such that

$$
(x, y) \in T^{t} \text { implies }\left(\lambda x, \lambda^{\alpha} y\right) \in T^{t}, \quad \forall \lambda>0 .
$$

This is a general concept of returns to scale that can deal with different degrees of scale: 1) $\alpha=1$ implies constant returns to scale; 2) $\alpha>1$ implies strictly increasing returns to scale; 3) $0<\alpha<1$ implies strictly decreasing returns to scale.

Throughout this paper, we allow a scalar $\alpha$ to vary with time. Thus, strictly speaking, it is more appropriate to use $\alpha^{t}$ rather than $\alpha$. However, we continue to use $\alpha$ to simplify the notation.

By using an equivalent representation of the technology by the input set $L^{t}(y)$, we can express the same condition as follows:

$$
L^{t}(\lambda y)=\lambda^{1 / \alpha} L^{t}(y), \quad \forall \lambda>0 \text { and } \forall y \in \mathbb{R}_{+}^{M}
$$

As Eq. (15) shows, when technical change is Hicks input neutral, the input set $L^{t}(y)$ can be decomposed into the time independent input set $\bar{L}(y)$ and the technology parameter $1 / A(t)$. We can draw the following implication of the $\alpha$-returns to scale for $\bar{L}(y)$, which we will use later:

Lemma 1: When the technology satisfies $\alpha$-returns to scale, the time independent input set $\bar{L}(y)$ defined in Eq. (15) satisfies the following relation:

$$
\bar{L}(\lambda y)=\lambda^{1 / \alpha} \bar{L}(y), \quad \forall \lambda>0 \text { and } \forall y \in \mathbb{R}_{+}^{M}
$$

Proof of Lemma 1: Applying Eq. (15) to $L(\lambda y)$ and $L(y)$ in Eq. (20), and cancelling $A(t)$ on both sides, we get Eq. (21). Q.E.D.

While input homotheticity and Hicks input neutrality are originally formulated by using the input set, it is possible to provide alternative definitions by using the input distance function. Similarly, as Färe and Mitchell (1993) first show, it is also possible to define $\alpha$-returns to scale by adopting the input distance function, as follows:

\footnotetext{
${ }^{21}$ For the same concept, Aczél (1966) uses the term 'almost homogeneous' and Lau (1978) and Färe and Mitchell (1993) use the term 'homogeneous of degree $\alpha$ '. We follow Boussemart et al. (2009), who first formulate this notion by using the technology set $T^{t}$ and call it ' $\alpha$-returns to scale' by showing that this concept could incorporate various types of returns to scale.
} 
Proposition 5 (Färe and Mitchell, 1993): The technology satisfies $\alpha$-returns to scale if and only if the input distance function satisfies the following equation:

$$
D_{i}^{t}(\lambda y, x)=\lambda^{-1 / \alpha} D_{i}^{t}(y, x), \quad \forall \lambda>0 \text { and } \forall(x, y) \in \mathbb{R}_{+}^{N+M}
$$

By using an equivalent representation of the technology by the input set $L^{t}(y)$, we can express the same condition as follows:

$$
P^{t}(\lambda x)=\lambda^{\alpha} P^{t}(x), \quad \forall \lambda>0 \text { and } \forall x \in \mathbb{R}_{+}^{N}
$$

As Eq. (17) shows, when technical change is Hicks output neutral, the output set $P^{t}(x)$ becomes the time independent output set $\bar{P}(x)$ multiplied by the technology parameter $B(t)$. We can also draw the following implication of the $\alpha$-returns to scale for $\bar{P}(x)$, which we will use later:

Lemma 2: When the technology satisfies $\alpha$-returns to scale, the time independent output set $\bar{P}(x)$ defined by Eq. (17) satisfies the following equation:

$$
\bar{P}(\lambda x)=\lambda^{\alpha} \bar{P}(x), \quad \forall \lambda>0 \text { and } \forall x \in \mathbb{R}_{+}^{N} .
$$

Proof of Lemma 2: Applying Eq. (17) to $L(\lambda y)$ and $L(y)$ in Eq. (23), and cancelling $B(t)$ on both sides yields Eq. (24). Q.E.D.

While output homotheticity and Hicks output neutrality are originally formulated by using the output set, it is also possible to provide equivalent definitions based on the output distance function, as summarized in the following Proposition.

Proposition 6: The technology satisfies $\alpha$-returns to scale if and only if the output distance function satisfies the following equation:

$$
D_{o}^{t}(\lambda x, y)=\lambda^{-\alpha} D_{o}^{t}(x, y), \quad \forall \lambda>0 \text { and } \forall(x, y) \in \mathbb{R}_{+}^{N+M}
$$

Proof of Proposition 6: First, suppose that Eq. (25) is satisfied. Then, the definition of $P^{t}(x)$ implies the following equation.

$$
\begin{gathered}
P^{t}(\lambda x)=\left\{y: D_{o}^{t}(\lambda x, y) \leq 1\right\} \\
\quad=\left\{y: \lambda^{-\alpha} D_{o}^{t}(x, y) \leq 1\right\}
\end{gathered}
$$

from Eq. (25), 


$$
=\left\{y: D_{o}^{t}\left(x, \lambda^{-\alpha} y\right) \leq 1\right\},
$$

since $D_{o}^{t}(x, y)$ is homogeneous of degree one in output $y$,

$$
\begin{gathered}
=\lambda^{\alpha}\left\{\lambda^{-\alpha} y: D_{o}^{t}\left(x, \lambda^{-\alpha} y\right) \leq 1\right\} \\
=\lambda^{\alpha} P(x) .
\end{gathered}
$$

Thus, we obtain Eq. (23), indicating the $\alpha$-returns to scale technology.

To show the converse, suppose that Eq. (23) is satisfied. Then, the definition of $D_{o}^{t}(x, y)$ implies the following equation.

$$
\begin{gathered}
D_{o}^{t}(\lambda x, y)=\inf \left\{\theta: y / \theta \in P^{t}(\lambda x)\right\} \\
=\inf \left\{\theta: y / \theta \in \lambda^{\alpha} P^{t}(x)\right\} \\
=\inf \left\{\theta: y /\left(\lambda^{\alpha} \theta\right) \in P^{t}(x)\right\} \\
=\lambda^{-\alpha} \inf \left\{\lambda^{\alpha} \theta: y /\left(\lambda^{\alpha} \theta\right) \in P^{t}(x)\right\} \\
=\lambda^{-\alpha} D_{o}^{t}(x, y) .
\end{gathered}
$$

from Eq. (23),

Thus, we obtain Eq. (25). QED.

Färe et al. (1985) show that input and output distance functions are reciprocal to each other, under constant returns to scale technology. Similarly, Boussemart et al. (2009) report the following relationship between two distance functions, under the $\alpha$-returns to scale technology. ${ }^{22}$ It allows one to compute the output distance function from the input distance function even when technology does not exhibit constant returns to scale.

Proposition 7 (Boussemart et al., 2009): The technology satisfies $\alpha$-returns to scale if and only if

$$
D_{o}^{t}(x, y)=\left(D_{i}^{t}(y, x)\right)^{-\alpha}, \forall(x, y) \in \mathbb{R}_{+}^{N+M}
$$

Proof of Proposition 7: First, suppose that Eq. (26) is satisfied. Then, the definition of $D_{o}^{t}(x, y)$ implies the following.

$$
\begin{aligned}
& D_{o}^{t}(\lambda x, y)=\lambda^{-\alpha}\left(D_{i}^{t}(y, x)\right)^{-\alpha} \\
& \text { since } D_{i}^{t}(y, x) \text { is homogeneous of degree one in input } x \\
& \quad=\lambda^{-\alpha} D_{o}^{t}(x, y)
\end{aligned}
$$

from Eq. (26).

\footnotetext{
${ }^{22}$ Boussemart et al. (2009) refer this result to Färe and Mitchell (1993). Nevertheless, Färe and Mitchell (1993) have not examined the relationship between input and output distance functions under $\alpha$-returns to scale such as Eq. (26). Thus, we provide the proof that Eq. (26) is the necessary and sufficient condition of $\alpha$-returns to scale technology for the first time. While an alternative proof of the sufficient condition is given by Mizobuchi (2017a), a full proof is given here for completeness.
} 
Thus, the technology is shown to satisfy $\alpha$-returns to scale.

To show the converse, suppose that the technology satisfies $\alpha$-returns to scale. Then, the definition of $D_{o}^{t}(x, y)$ implies the following.

$$
\begin{gathered}
D_{o}^{t}(x, y)=\inf \left\{\theta: D_{i}^{t}(y / \theta, x) \geq 1\right\} \\
=\inf \left\{\theta: \theta^{1 / \alpha} D_{i}^{t}(y, x) \geq 1\right\} \\
=\inf \left\{\theta: \theta \geq\left(D_{i}^{t}(y, x)\right)^{-\alpha}\right\} \\
=\left(D_{i}^{t}(y, x)\right)^{-\alpha} .
\end{gathered}
$$

from Eq. (22).

Thus, we obtain Eq. (26). QED.

Under the $\alpha$-returns to scale technology, the proportional increase in input by a factor of $\lambda$ induces the radial expansion of the output isoquant by a factor of $\lambda^{\alpha}$. As Lancaster (1968) attempts to do in the single output case, it is possible to consider an extension of this class of technology by adopting the more general function $f(\lambda)$ rather than $\lambda^{\alpha}$ to capture the impact on the output isoquant as follows:

Definition 7: The technology is generally homogeneous if and only if there is a nonconstant increasing function $f$ such that

$$
(x, y) \in T^{t} \text { implies }(\lambda x, f(\lambda) y) \in T^{t}, \quad \forall \lambda>0 .
$$

By using an equivalent representation of the technology by the output set $P^{t}(y)$, we can express the same condition as follows:

$$
P^{t}(\lambda x)=f(\lambda) P^{t}(x), \quad \forall \lambda>0 .
$$

When we sequentially apply Eq. (28), we obtain the following equation:

$$
f(\lambda \mu)=f(\lambda) f(\mu), \quad \forall \lambda>0 \text { and } \forall \mu>0 .
$$

This is a type of Cauchy functional equation, which arises in various fields of economics. Aczél (1966) finds the solutions to several Cauchy functional equations in different domains. 
Proposition 8 (Aczél, 1966): ${ }^{23}$ A non-constant increasing function $f$ satisfies Eq. (29) if and only if there is $\alpha>0$ such that

$$
f(\lambda)=\lambda^{\alpha}, \quad \forall \lambda>0
$$

It indicates that the generally homogeneous technology proposed by Eq. (27) turns out to equal to the $\alpha$-returns to scale technology defined by Eq. (19). Therefore, the latter type of technology is now shown to be much more general than it appears to be at first sight, by allowing the output isoquant to radially expand by a variety of rates in response to the proportional increase in input.

\section{Main Results}

First, we show that the two types of homotheticity conditions are equivalent under $\alpha$ returns to scale technology.

Proposition 9: Suppose the technology satisfies $\alpha$-returns to scale. The technology is output homothetic if and only if it is input homothetic.

Proof of Proposition 9: We first show that if the technology is input homothetic, then it is also output homothetic under the $\alpha$-returns to scale technology. We rearrange Eq. (11) as following:

$$
\begin{aligned}
\left(D_{i}^{t}(y, x)\right)^{-\alpha} & =\left(D_{i}^{t}\left(1_{M}, x\right)\right)^{-\alpha}\left(1 / H^{t}(y)\right)^{-\alpha} \\
& \text { by raising both side of Eq. (11) to the power of }-\alpha \\
D_{o}^{t}(x, y) & =D_{o}^{t}\left(x, 1_{M}\right)\left(H^{t}(y)\right)^{\alpha}
\end{aligned}
$$

from Eq. (26).

Note that Eq. (12) indicates that, output homotheticity means the output isoquant of $x$ is a proportional expansion of the isoquant of $1_{N}$. Thus, the ratio of the radial distance from the origin to the output isoquant of $x$ to the radial distance from the origin to the output isoquant of $1_{N}$ is $G^{t}(x)$, which only depends on $x$.

Since the radial distance from the origin to the output isoquant of $x$ is $1 / D_{o}^{t}(x, y)$, the radial distance from the origin to the output isoquant of $1_{N}$ is $1 / D_{o}^{t}\left(1_{N}, y\right)$. Therefore, by relying on Eq. (31), we can show that its ratio only depends on $x$ as follows:

$$
\frac{D_{o}^{t}(x, y)}{D_{o}^{t}\left(1_{N}, y\right)}=\frac{D_{o}^{t}\left(x, 1_{M}\right)\left(H^{t}(y)\right)^{\alpha}}{D_{o}^{t}\left(1_{N}, 1_{M}\right)\left(H^{t}(y)\right)^{\alpha}}=\frac{D_{o}^{t}\left(x, 1_{M}\right)}{D_{o}^{t}\left(1_{N}, 1_{M}\right)}
$$

From Eq. (32), we can derive the following equation:

\footnotetext{
${ }^{23}$ Strictly speaking, Aczél (1966) characterizes two function satisfying Eq. (29) such as $f(\lambda)=\lambda^{\alpha}$ for all $\lambda>0$ and $f(\lambda)=0$ for all $\lambda>0$. Since we focus on non-constant functions, we omit the latter case.
} 


$$
D_{o}^{t}(x, y)=\frac{D_{o}^{t}\left(1_{N}, y\right)}{D_{o}^{t}\left(1_{N}, 1_{M}\right) / D_{o}^{t}\left(x, 1_{M}\right)}
$$

Let $G^{t}(x)=D_{o}^{t}\left(1_{N}, 1_{M}\right) / D_{o}^{t}\left(x, 1_{M}\right)$. It is immediate that $G^{t}(x)$ satisfies the properties B.1-B.5. Thus, we obtain Eq. (13).

We similarly show that if the technology is output homothetic, then it is also input homothetic under the $\alpha$-returns to scale technology. We rearrange Eq. (13) as follows:

$$
\left(D_{o}^{t}(x, y)\right)^{-1 / \alpha}=\left(D_{o}^{t}\left(1_{N}, y\right)\right)^{-1 / \alpha}\left(1 / G^{t}(x)\right)^{-1 / \alpha}
$$

by raising both side of Eq. (13) to the power of $-1 / \alpha$,

$$
D_{i}^{t}(y, x)=D_{i}^{t}\left(y, 1_{N}\right)\left(G^{t}(x)\right)^{1 / \alpha}
$$

from Eq. (26).

Note that Eq. (10) indicates that, input homotheticity means the input isoquant of $y$ is a proportional expansion of the isoquant of $1_{M}$. Thus, the ratio of the radial distance from the origin to the input isoquant of $y$ to the radial distance from the origin to the input isoquant of $1_{M}$ is $H^{t}(y)$, which only depends on $y$.

Since the radial distance from the origin to the input isoquant of $y$ is $1 / D_{i}^{t}(y, x)$, the radial distance from the origin to the input isoquant of $1_{M}$ is $1 / D_{i}^{t}\left(1_{M}, x\right)$. Therefore, by relying on Eq. (34), we can show that its ratio only depends on $y$ as follows:

$$
\frac{D_{i}^{t}(y, x)}{D_{i}^{t}\left(1_{M}, x\right)}=\frac{D_{i}^{t}\left(y, 1_{N}\right)\left(G^{t}(x)\right)^{1 / \alpha}}{D_{i}^{t}\left(1_{M}, 1_{N}\right)\left(G^{t}(x)\right)^{1 / \alpha}}=\frac{D_{i}^{t}\left(y, 1_{N}\right)}{D_{i}^{t}\left(1_{M}, 1_{N}\right)}
$$

From Eq. (35), we can derivate the following equation:

$$
D_{i}^{t}(y, x)=\frac{D_{i}^{t}\left(1_{M}, x\right)}{\left(D_{i}^{t}\left(1_{M}, 1_{N}\right) / D_{i}^{t}\left(y, 1_{N}\right)\right)} .
$$

Let $H^{t}(y)=D_{i}^{t}\left(1_{M}, 1_{N}\right) / D_{i}^{t}\left(y, 1_{N}\right)$. It is immediate that $H^{t}(y)$ satisfies the properties A.1-A.5. Thus, we obtain Eq. (11). QED.

Second, we show that two types of Hicks neutrality conditions are also equivalent under $\alpha$-returns to scale technology.

Proposition 10: Suppose the technology satisfies $\alpha$-returns to scale. The technical change is Hicks input neutral if and only if it is Hicks output neutral.

Proof of Proposition 10: We first show that if the technical change is Hicks input neutral, then it is also Hicks output neutral under the $\alpha$-returns to scale technology. We rearrange Eq. (16) as follows:

$$
D_{i}^{t}(y, x)=\bar{D}_{i}(y, A(t) x),
$$


because $\bar{D}_{i}(y, x)$ is homogeneous of degree one in input $x$, $\left(D_{i}^{t}(y, x)\right)^{-\alpha}=\left(\bar{D}_{i}(y, A(t) x)\right)^{-\alpha}$

by raising both side of the above equation to the power $-\alpha$,

$$
\begin{gathered}
D_{o}^{t}(y, x)=\bar{D}_{o}(A(t) x, y) \\
D_{o}^{t}(y, x)=\bar{D}_{o}(x, y) /(A(t))^{\alpha}
\end{gathered}
$$

from Eq. (25).

Thus, we obtain Eq. (18).

We similarly show that if the technical change is Hicks output neutral, then it is also Hicks input neutral under the $\alpha$-returns to scale technology. We rearrange Eq. (18) as follows:

$$
\begin{gathered}
D_{o}^{t}(x, y)=\bar{D}_{o}(x, y / B(t)), \\
\text { because } \bar{D}_{o}(x, y) \text { is homogeneous of degree one in output } y, \\
\left(D_{o}^{t}(x, y)\right)^{-1 / \alpha}=\left(\bar{D}_{o}(x, y / B(t))\right)^{-1 / \alpha} \\
\text { by raising both side of the above equation to the power }-1 / \alpha, \\
D_{i}^{t}(y, x)=\bar{D}_{i}(y / B(t), x) \\
D_{i}^{t}(y, x)=(B(t))^{1 / \alpha} \bar{D}_{i}(y, x)
\end{gathered}
$$

from Eq. (22).

Thus, we obtain Eq. (16). QED.

Now, we can schematically show the relationship between input and output homotheticity and different returns to scale technologies by Figure 1. We emphasize two points. First, the sets of input and output homothetic technology overlap, when we focus on $\alpha$-returns to scale technology. Since the set of constant returns to scale technology is a subset of the set of $\alpha$-returns to scale technology, when we are concerned with constant returns to scale technology, the two sets also overlap. Second, the sets of input and output homothetic technology may overlap outside the set of $\alpha$ returns to scale technology. It means that even if the technology does not satisfy $\alpha$ returns to scale, the technology might be input and output homothetic at the same time. We can also regard Figure 1 as the relationship between input and output Hicks neutrality and different returns to scale technologies as well. The same explanation for input and output homotheticity applies to Hicks input and output neutrality. 


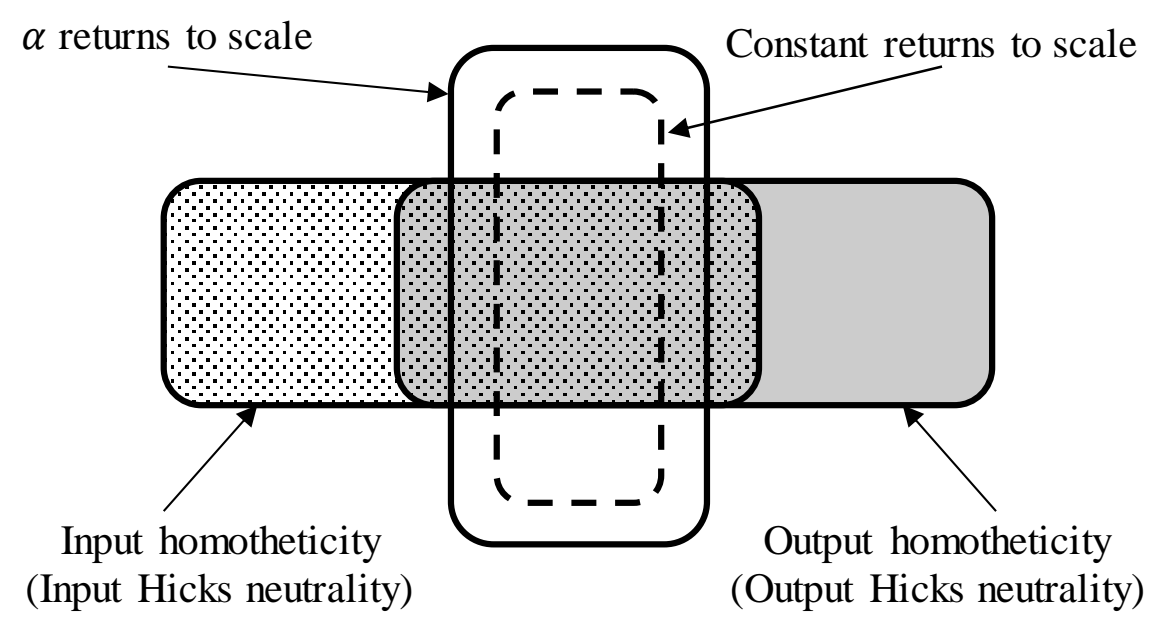

Figure 1: Relationship between different conditions of technology

Inverse homotheticity is equivalent to simultaneous input and output homotheticity. Since one type of homotheticity implies the other under constant returns to scale, it is not necessary to assume both conditions simultaneously. Thus, by using Proposition 9, we can strengthen Proposition 2 as follows:

Corollary 1: The period $t$ Malmquist productivity index $M P I^{t}$ equals the period $t$ Hicks-Moorsteen productivity index $H M P I^{t}$ if and only if the technology is input homothetic (or output homothetic) and exhibits constant returns to scale.

Since input and output homothetic technologies are equivalent under constant returns to scale technology, the relationship that holds when the technology exhibits output homotheticity and constant returns to scale also holds when it exhibits input homotheticity and constant returns to scale. Thus, we can draw the following corollary as the immediate implication from Proposition 3 and Proposition 9.

Corollary 2: The Malmquist productivity index MPI equals the Hicks-Moorsteen productivity index HMPI if the technology is input homothetic and exhibits constant returns to scale.

Since Hicks input and output neutrality are equivalent under constant returns to scale technology, the relationship that holds when the technology exhibits constant returns to scale and the technical change is Hicks output neutral also holds when the technology exhibits constant returns to scale and the technical change is Hicks input neutral. Thus, we can draw the following corollary as the immediate implication from Proposition 4 and Proposition 10.

Corollary 3: The Malmquist productivity index MPI equals the Hicks-Moorsteen productivity index HMPI if the technology exhibits constant returns to scale and the technical change is Hicks input neutral. 


\section{Conclusion}

In this paper, we deal with $\alpha$-returns to scale technology. This is a general homogeneous technology, which allows the technology to exhibit decreasing, constant and increasing returns to scale. While there exist two types of conditions for the homothetic technology as well the Hicks neutral technical change in the multiple output case, we show that they are equivalent when the technology satisfies $\alpha$ returns to scale.

In turn, this means that as long as we deal with $\alpha$-returns to scale technology, it is not necessary to impose two types of homotheticity and Hicks neutrality at the same time. For example, under $\alpha$-returns to scale technology, if the technology is input homothetic, it is necessarily output homothetic, and vice versa. Similarly, under the same condition, if the technical change is Hicks input neutral, it is necessarily Hicks output neutral, and vice versa. Thus, this paper strengthens some existing theoretical results by reducing the number of conditions that need to be satisfied, or by deriving the same relationship under alternative conditions. ${ }^{24}$

It is worth noting that there exist different types of homotheticity and Hicks neutrality other than the one we deal with in this paper. Our result is not necessarily applicable to all those. For example, input and output scale homothetic technologies and the implicit Hicks input and output technical changes are not equivalent even when the technology satisfies $\alpha$-returns to scale. ${ }^{25}$ What are the necessary restrictions on technology to guarantee the equivalence between these conditions? This question remains open.

The present paper shows that $\alpha$-returns to scale is a sufficient condition for the equivalence between input-isoquant-based and output-isoquant-based conditions for homotheticity and Hicks neutrality. Thus, it does not exclude the possibility that one type of condition implies the other, even when the technology does not satisfy $\alpha$-returns to scale. The characteristics of technology where two types of conditions are equivalent are not fully identified. We leave the search for the necessary condition for the equivalence between input-isoquant-based conditions and output-isoquant-based conditions for future research.

Another interesting question for future research is to look at the implications of a more general structure than the $\alpha$-returns to scale technology, e.g., when $(x, y) \in$ $T^{t}$ implies $\left(\lambda^{\alpha_{1}} x_{1}, \ldots, \lambda^{\alpha_{M}} x_{M}, \lambda y\right) \in T^{t}$ for all $\lambda>0$ (see Aczél (1966) and Lau (1978) for related discussions). Finally, it would be also interesting to explore the relationship between homotheticity and Hicks neutrality in the sense of when one implies the other, e.g., similarly as was explored by Sato and Ramachandran (1998, p. 24) for the context of local (or derivate-based) returns to scale analysis.

\footnotetext{
${ }^{24}$ Primont and Primont (1994) propose a test for input as well as output homotheticity. As long as we are concerned with $\alpha$-returns to scale technology, we do not need to test both input and output homotheticity. When one is satisfied, the other is automatically satisfied.

${ }^{25}$ See Zelenyuk (2014) for scale homotheticity.
} 


\section{Reference}

Aczél, J., (1966). Lectures on Functional Equations and Their Applications, New York: Academic Press.

Balk, B.M., Färe. R., Grosskopf, S. and Margaritis, D., (2008). "Exact Relations between Luenberger Productivity Indicators and Malmquist Productivity Indexes." Economic Theory, Vol.35, No.1, pp.187-190.

Balk, B.M., (1998). Industrial Price, Quantity, and Productivity Indices: The MicroEconomic Theory and an Application, Boston, MA: Springer US.

Bjurek, H., (1996). “The Malmquist Total Factor Productivity Index.” Scandinavian Journal of Economics, Vol.98, No.2, pp.303-313.

Blackorby, C., Lovell, C.A.K. and Thursby, M.C., (1976). "Extended Hicks Neutral Change.” Economic Journal, Vol.86, No.344, pp.845-852.

Boussemart, J.P., Briec, W., Peypoch, N. and Tavéra C., (2009). “ $\alpha$-Returns to Scale and Multi-Output Production Technologies.” European Journal of Operational Research, Vol.197, No.1, pp.332-339.

Briec, W. and Kerstens, K., (2004). “A Luenberger-Hicks-Moorsteen Productivity Indicator: its Relation to the Hicks-Moorsteen Productivity Index and the Luenberger Productivity Indicator." Economic Theory, Vol.23, No.4, pp.925939.

Caves, D.W., Christensen, L.R. and Diewert, W.E., (1982). "The Economic Theory of Index Numbers and the Measurement of Input, Output, and Productivity." Econometrica, Vol.50, No.6, pp.1393-1414.

Chambers, R.G., (2002). "Exact Nonradial Input, Output, and Productivity Measurement." Economic Theory, Vol.20, No.4, pp.751-765.

Chambers, R.G. and Färe, R., (1994). "Hicks' Neutrality and Trade Biased Growth: A Taxonomy." Journal of Economic Theory, Vol.64, pp.554-567.

Diewert, W.E., (1992). "Fisher Ideal Output, Input, and Productivity Indexes Revisited." Journal of Productivity Analysis, Vol.3, No.3, pp.211-248.

Färe, R., Grosskopf, S. and Lovell, C.A.K., (1985). The Measurement of Efficiency of Production, Boston: Kluwer Academic Publishers.

Färe, R., Grosskopf, S. and Roos, P., (1996). "On Two Definitions of Productivity." Economics Letters, Vol.53, No.3, pp.269-274.

Färe, R. and Mitchell, T., (1993). "Multiple Outputs and Homotheticity." Southern Economic Journal, Vol.60, No.2, pp.287-296.

Färe, R. and Primont, D., (1995). Multi-Output Production and Duality: Theory and Applications, Boston, MA: Kluwer Academic Publishers.

Hicks, J.R., (1932). The Theory of Wages, London: Macmillan.

Jacobsen, S.E., (1970). "Production Correspondences." Econometrica, Vol.38, No.5, p.754.

Lancaster, K., (1968). Mathematical Economics, New York, NY: Macmillan.

Lau, L.J., (1978). “Application of Profit Functions.” In M. Fuss and D. McFadden, eds. Production Economics: A Dual Approach to Theory and Applications 
Volume I: The Theory of Production. Amsterdam: North-Holland, pp. 133-216.

Mizobuchi, H., (2017a). "A Superlative Index Number Formula for the HicksMoorsteen Productivity Index." Journal of Productivity Analysis, Vol.48, No.23, pp.167-178.

Mizobuchi, H., (2017b). "Productivity Indexes under Hicks Neutral Technical Change." Journal of Productivity Analysis, Vol.48, No.1, pp.1-9.

Primont, Daniel and Primont, Diane, (1994). "Homothetic Non-Parametric Production Models.” Economics Letters, Vol.45, No.2, pp.191-195.

Sato, R. and Ramachandran, R. V., (1998). Symmetry and Economic Invariance: An Introduction, Boston, MA: Springer US.

Shephard, R.W., (1953). Cost and Production Functions, Princeton, NJ: Princeton University Press.

Shephard, R.W., (1970). Theory of Cost and Production Function, Princeton, NJ: Princeton University Press.

Sickles, R.C. and Zelenyuk, V., (2018). Measurement of Productivity and Efficiency: Theory and Practice, New York, NY: Cambridge University Press.

Zelenyuk, V., (2014). "Scale Efficiency and Homotheticity: Equivalence of Primal and Dual Measures." Journal of Productivity Analysis, Vol.42, No.1, pp.15-24. 